\title{
Albayzín-2014 evaluation: audio segmentation and classification in broadcast news domains
}

\author{
Diego Castán ${ }^{*}$, David Tavarez² ${ }^{2}$ Paula Lopez-Otero ${ }^{3}$, Javier Franco-Pedroso ${ }^{4}$, Héctor Delgado 5 , \\ Eva Navas ${ }^{2}$, Laura Docio-Fernández ${ }^{3}$, Daniel Ramos ${ }^{4}$, Javier Serrano ${ }^{5}$, Alfonso Ortega ${ }^{1}$ and Eduardo Lleida ${ }^{1}$
}

\begin{abstract}
Audio segmentation is important as a pre-processing task to improve the performance of many speech technology tasks and, therefore, it has an undoubted research interest. This paper describes the database, the metric, the systems and the results for the Albayzín-2014 audio segmentation campaign. In contrast to previous evaluations where the task was the segmentation of non-overlapping classes, Albayzín-2014 evaluation proposes the delimitation of the presence of speech, music and/or noise that can be found simultaneously. The database used in the evaluation was created by fusing different media and noises in order to increase the difficulty of the task. Seven segmentation systems from four different research groups were evaluated and combined. Their experimental results were analyzed and compared with the aim of providing a benchmark and showing up the promising directions in this field.
\end{abstract}

Keywords: Audio segmentation, Broadcast news, Albayzín-2014 evaluation

\section{Introduction}

Automatic audio segmentation aims at providing boundaries to delimit portions of audio with homogeneous acoustic content. The resulting segments are classified in different acoustic types according to the final application, such as different speakers, languages, speech/nonspeech portions, or acoustic events among others. In most cases, automatic audio segmentation is considered a pre-processing tool to improve the performance of the subsequent system related with speech technologies. For example, in very large multimedia repositories, the speech is usually found along with music or environmental noise. The presence of these acoustic classes must be accurately labeled because it is critical for the subsequent systems to be successful. Thus, the development of accurate Audio Segmentation Systems is essential to allow postprocessing systems, such as automatic speech recognition (ASR) or spoken document retrieval (SDR), to perform adequately in real-world environments.

*Correspondence: dcastan@unizar.es

1 ViVoLab, Universidad de Zaragoza, Zaragoza, Spain

Full list of author information is available at the end of the article
Audio segmentation systems can address the problem in different fields or contexts. In the first works of automatic segmentation, the goal was the challenging segmentation of sports material and commercials. The studies focused on speech/music segmentation from radio stations as in [1] and [2] showing the importance of the audio segmentation to improve ASR systems. The following studies dealt with the recognition of broad classes to produce an adaptation of the ASR models. For example, Srinivasan [3] classified the audio of a video into mixed classes such as music with speech or speech with background noise using a combination of acoustic and perceptual features. Nowadays, most of the studies focus on the robust and generic segmentation of broad classes [4] and the segmentation of acoustic events [5] for audio retrieval in large multimedia databases.

A specific task with large multimedia databases is the segmentation of broadcast news (BN) recordings. This task is very challenging because the audio contains different kinds of sequences with a very heterogeneous style. Several international evaluation campaigns, such as the TREC NIST evaluations for SDR [6], the ESTER evaluation campaigns for rich transcription (RT) in French [7], and the COST278 evaluation for segmentation and

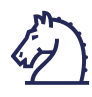

C 2015 Castán et al. Open Access This article is distributed under the terms of the Creative Commons Attribution 4.0 International License (http://creativecommons.org/licenses/by/4.0/), which permits unrestricted use, distribution, and reproduction in any medium, provided you give appropriate credit to the original author(s) and the source, provide a link to the Creative Commons license, and indicate if changes were made. 
speaker clustering in a multi-lingual domain [8], have already been proposed to face this task in the past.

Nowadays, the amount of audio documents is exponentially increasing due to the audio-sharing websites or the audio-on-demand systems. Users around the world can upload and share their contents and, for that reason, the variability of the acoustic conditions is extremely high. As a result, systems must be able to adapt their role in high-variability data spaces, providing robust performance in different conditions. Due to the importance of audio segmentation and the need to develop robust systems capable of operating over a rich variety of audio conditions, the Albayzín-2014 campaign was proposed as an international evaluation to measure the performance of segmentation systems for different databases and different contexts. This segmentation evaluation, which is part of an open set of evaluations organized by the $\mathrm{RTTH}^{1}$ every 2 years, compares systems and approaches from different research institutions in an independent way.

In contrast to previous evaluations such as Albayzín2010 [9], where five unambiguous acoustic classes were defined, the Albayzín-2014 evaluation proposed the delimitation of the presence of speech, music and/or noise that can be found simultaneously. Another relevant difference was the composition of the database: while in previous evaluations the databases were composed of a unique BN media (TV in Albayzín-2010 mostly in Catalan [9] or radio in Albayzín-2012 [10] mostly in Spanish), the Albayzín-2014 database was a combination and fusion of three different databases with TV, radio, and noise recordings. This composition increased the difficulty of the task since the resulting database introduced more variability, presenting more realistic conditions over a wide variety of acoustic sources.

The remainder of the paper is organized as follows: the database and the metric used for Albayzín-2014 segmentation evaluation are presented in Section 2. Section 3 briefly describes the submitted systems. The results of the evaluation and the fusion of the systems are presented and discussed in Section 4. Finally, the summary and the conclusions are presented in Section 5.

\section{Database and evaluation metric}

The proposed evaluation consisted of segmenting a broadcast audio document and assigning labels for each segment indicating the presence of speech, music, and/or noise. That is, two or more classes could be found simultaneously in audio segments and the goal was to indicate if one, two, or the three aforementioned classes were present for a given time instant. For example, music could be overlapped with speech, or noise could be found in the background when someone was speaking. Therefore, the presence of these three classes involve the definition of eight non-overlapping classes: silence, speech, music, noise, speech with music, speech with noise, music with noise, and speech with music and noise. In this evaluation, Speech was present every time that a person was speaking but not in the background or singing. Music was understood in a general sense and noise was considered every time some acoustic content was present different than speech and music (including speech in the background, which usually comes from a crowd).

The goal was to segment and label audio documents indicating where speech, music, and/or noise were present. Unlike 2010 evaluation criteria [9], no prior classes were defined (speech, music, speech with noise in background, speech with music in background, other) and a multiple layer labeling approach was proposed instead. In summary, the goal was to segment the incoming audio into three (possibly overlapped) acoustic classes: speech, music, and noise, where the audio was drawn from different databases that have been merged or even overlapped, thus dramatically increasing the difficulty of the task with regard to previous evaluations.

\subsection{Database}

The database for this evaluation is a combination of three databases defined below:

The first dataset is the Catalan broadcast news database from the 3/24 TV channel proposed for the Albayzín-2010 Audio Segmentation Evaluation [9]. This database was recorded by the TALP Research Center of the Polytechnic University of Catalonia in 2009 under the Tecnoparla project [11] funded by the Government of Catalonia. The Corporació Catalana de Mitjans Audiovisuals (CCMA), owner of the multimedia content, allows its use for technology research and development. The database consists of around $87 \mathrm{~h}$ of recordings in which speech can be found $92 \%$ of the time, music is present $20 \%$ of the time, and noise in the background is present $40 \%$ of the time. Another class called others was defined which can be found $3 \%$ of the time. Regarding the overlapped classes, speech can be found along with noise $40 \%$ of the time and along with music $15 \%$ of the time.

The second dataset is the Aragón Radio database from the Corporación Aragonesa de Radio y Televisión (CARTV) which was used for the Albayzín-2012 Audio Segmentation Evaluation [10]. As the owner of the audio content, Aragón Radio and the Corporación Aragonesa de Radio y Televisión allow the use of these data for research purposes.

The last dataset is composed of environmental sounds from Freesound.org [12] and HuCorpus [13]. These sounds were merged with segments from the $3 / 24 \mathrm{TV}$ and Aragón Radio databases.

All the data was supplied in PCM format, mono, little endian 16 bit resolution, and $16 \mathrm{kHz}$ sampling frequency. The database includes approximately $35 \mathrm{~h}$ of audio: $20 \mathrm{~h}$ 


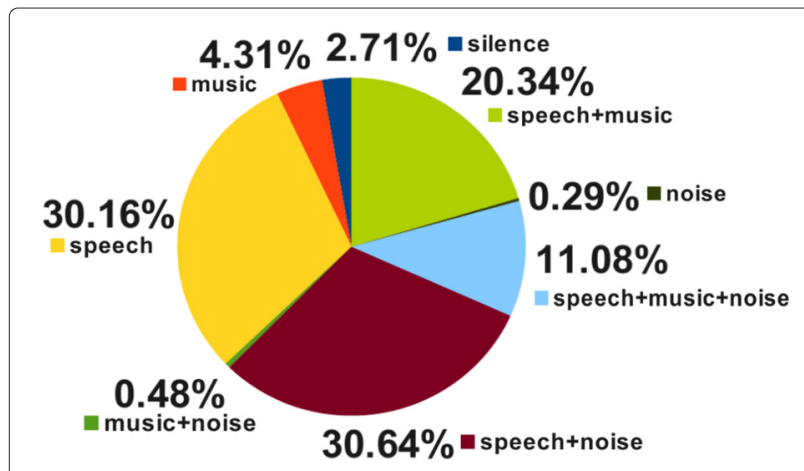

Fig. 1 Distribution of the different audio classes in the training set of the Albayzín-2014 evaluation

were used for the training set and $15 \mathrm{~h}$ were used for the test set. The distribution of the audio classes in the training set is presented in Fig. 1. The chart shows that the classes containing speech represent more than $92 \%$ of the total time. There are two residual classes, isolated noise and music with noise, that represent less than 0.5 and $0.3 \%$ of the total time, respectively.

\subsection{Evaluation metric}

As in the NIST RT Diarization evaluations [14], the segmentation error score (SER) was used to measure the performance of the proposed systems. SER is computed as the fraction of class time that is not correctly attributed to that specific class (speech, noise, or music). The SER score was computed over the entire file to be processed, including regions where more than one class was present (overlap regions).

The overall SER score is defined as the ratio of the overall segmentation error time to the sum of the durations of the segments that are assigned to each class in the file.

Given the dataset to evaluate $\Omega$, each document is divided into contiguous segments at all "class change points" which occur each time any reference class (oracle) or system class (hypothesis) starts or ends. Thus, the set of active reference classes and/or system classes does not change during any segment. The segmentation error time for each segment $n$ is defined as

$$
E(n)=T(n)\left[\max \left(N_{\text {ref }}(n), N_{\text {sys }}(n)\right)-N_{\text {Correct }}(n)\right]
$$

where $T(n)$ is the duration of segment $n, N_{\text {ref }}(n)$ is the number of reference classes that are present in segment $n$, $N_{\text {sys }}(n)$ is the number of system classes that are present in segment $n$, and $N_{\text {Correct }}(n)$ is the number of reference classes in segment $n$ correctly assigned by the segmentation system.

$$
\mathrm{SER}=\frac{\sum_{n \in \Omega} E(n)}{\sum_{n \in \Omega}\left(T(n) N_{\mathrm{ref}}(n)\right)}
$$

The segmentation error time includes the amount of time that is assigned to the wrong class, missed class time, and false alarm class time:

- Class error time: The class error time is the amount of time that has been assigned to an incorrect class. This error can occur in segments where the number of system classes is greater than the number of reference classes but also in segments where the number of system classes is lower than the number of reference classes whenever the number of system classes and the number of reference classes are greater than zero.

- Missed class time: The missed class time refers to the amount of time that a class is present but not labeled by the segmentation system in segments where the number of system classes is lower than the number of reference classes.

- False alarm class time: The false alarm class time is the amount of time that a class has been labeled by the segmentation system but is not present in segments where the number of system classes is greater than the number of reference classes.

The forgiveness collar defines a no-score area around reference segment boundaries. Typically, the collar is 250 $\mathrm{ms}$ for speaker diarization tasks [14] and $1 \mathrm{~s}$ for segmentation tasks [9]. A forgiveness collar of $1 \mathrm{~s}$, before and after each reference boundary, was considered in order to take into account both inconsistent human annotations and the uncertainty about when a class begins or ends. This collar is enough for the purpose of this segmentation task where the goal is to identify the areas with speech and their background to allow the adaptation of models for other systems as ASR. The implementation of the collar was provided by scoring tool of the NIST RT Diarization evaluations [14].

\section{Segmentation systems}

\subsection{General description of audio segmentation systems}

The general scheme of an audio segmentation system can be divided into two basic steps: the feature extraction method and the segmentation/classification strategy. Lavner in [15] and more recently Theodorou in [16] provide good reviews of the features and the classification methods used in the literature.

The acoustic feature extraction is the first step in an audio segmentation system. The audio input is divided into overlapping windows and, for each window, a feature vector is extracted. The feature vectors are descriptors used to distinguish the differences among classes in the time and frequency domains. Features can be grouped into two classes according to the time span they represent: frame-based and segment-based. Frame-based features are extracted within short periods of time (between 
10 and $30 \mathrm{~ms}$ ) and are commonly used in speech-related tasks where the signal can be considered stationary over that frame. Mel Frequency Cepstrum Coefficients (MFCC) or Perceptual Linear Prediction (PLP) coefficients are generally used as frame-based features as proposed in [17-22] among a great collection of works. Frame-based features have also been proposed for segmenting and classifying $\mathrm{BN}$ audio into broad classes. As an example, two pitchdensity-based features are proposed in [23], the authors use short-time energy (STE) in [1,24, 25], and harmonic features are used in [26-28]. The frame-based features can be directly used in the classifier. However, some classes are better described by the statistics computed over longer periods of time (from 0.5 to $5 \mathrm{~s}$ long). These characteristics are referred in the literature as segment-based features $[29,30]$. For example, in [31], a content-based speech discrimination algorithm is designed to exploit the long-term information inherent in the modulation spectrum; and in [32], authors propose two segment-based features: the variance of the spectrum flux (VSF) and the variance of the zero crossing rate (VZCR).

Once the feature vectors are computed, the next step deals with the detection and the classification of the segments. The segmentation/classification strategies can be divided into two different groups depending on how the segmentation is performed. The first group detects the break-points in a first step and then classifies each delimited segment in a second step. We refer to them as segmentation-and-classification approaches but they are also known in the literature as distance-based techniques. These algorithms have the advantage that they do not need labels to delimit the segments because the segmentation is based on a distance metric estimated for adjacent segments. When the distance between two adjacent segments is greater than a certain threshold, a break-point is set and identified as an acoustic changepoint. The resulting segments are clustered or classified in a second stage. The Bayesian Information Criterion (BIC) is a well-known distance-based algorithm. It is widely employed in many studies, such as [33], to generate a break-point for every speaker or environment/channel condition change in the BN domain and also, in [34] and [35], to identify mixed-language speech and speaker changes, respectively. The second group of segmentation/classification strategies is known as segmentation-byclassification or model-based segmentation. In contrast to the segmentation-and-classification algorithms, these algorithms classify consecutive fixed-length audio segments and, therefore, segment labels are required in a training step because each class of interest is described by a model. The segmentation is produced directly by the classifier as a sequence of decisions. This sequence is usually smoothed to improve the segmentation performance, since the classification of frames produces some spurious labels because adjacent frames are poorly considered.

A good and common approach to this procedure can be found in [36] where the author combines different features with a Gaussian Mixture Model (GMM) and a maximum entropy classifier. In [37], the authors use a factor analysis approach to adapt a universal GMM model to classify BN in five different classes. The final decisions of both systems are smoothed with a Hidden Markov Model (HMM) to avoid sudden changes.

Both segmentation/classification strategies were used by participants in the Albayzín-2014 Audio Segmentation Evaluation: three participating groups chose segmentation-by-classification algorithms with different model strategies and one participating group chose a segmentation-and-classification algorithm based on BIC for the first stage and on different classification systems for the second stage. A brief description of the features and the systems is given below.

\subsection{Description of the participating systems}

Four research groups participated in this evaluation with seven different systems: Aholab-EHU/UPV (University of the Basque Country), GTM-UVigo (University of Vigo), ATVS-UAM (Autonomous University of Madrid), and CAIAC-UAB (Autonomous University of Barcelona). Each participant had 3 months to design the segmentation system with the training data. After that time, participants were given 1 month to process the test data. The participants had to submit their results with hard-segmentation labels (in RTTM format from NIST) along with a technical description of the submitted systems. All participant teams had to submit at least a primary system but they could also submit up to two contrastive systems. Also, for fusion purposes, participants were required to submit the frame-level scores for each non-overlapping audio class. Groups are listed in the order in which their primary systems were ranked in the evaluation. A more detailed description of the systems can be found in the Advances in Speech and Language Technologies for Iberian Languages proceedings [38].

\subsubsection{Group 1}

This group presented a single primary system where two different segmentation-by-classification strategies were fused to build a robust system.

The first strategy consisted of a hidden Markov model (HMM) scheme with eight separate HMM models for each non-overlapping class: silence, speech, music, noise, speech with music, speech with noise, music with noise, and speech with music and noise. Thirteen MFCCs with first and second derivatives were used for the classification and each HMM had 3 states with 512 Gaussian components per state. 
The second strategy consisted of a GMM presegmentation and a speech label refinement by means of ivector classification via multilayer perceptron (MLP). Six GMMs with 32 components for silence, music, noise, clean speech, speech with noise, and speech with music were used in a Viterbi segmentation. Twelve MFCCs with first- and second-order derivatives were used for the classification (the energy-related coefficient was not used in this case). Once the speech segments were identified, the i-vector extraction process was carried out. A sliding window was used to extract the i-vectors corresponding to each speech segment. Then, an MLP was used to classify each i-vector as clean speech, speech with noise, speech with music, or speech with music and with noise.

The outputs of both subsystems were post-processed to discard too short segments. Finally, a label fusion algorithm based on the confusion matrices of the systems involved in the fusion was applied to combine the results of both subsystems and maximize the precision of the final labels.

\subsubsection{Group 2}

Group 2 presented a primary system and two contrastive systems, all of them with a segmentation-andclassification strategy.

The segmentation stage was common for all the systems and consisted of a Bayesian Information Criterion (BIC) approach using 12 MFCCs plus energy and featuring a false alarm rejection strategy: the occurrence of acoustic change-points was supposed to follow a Poisson process, and a change-point was discarded with a probability that varied in function of the expected number of occurrences in the time interval going from the previous change-point to the candidate change-point.

The classification stage was different for each system. The primary system was developed using i-vector representations of the segments obtained from the previous step with logistic regression classification. Perceptual linear prediction (PLP) analysis was used to extract 13 cepstral coefficients, which were combined with two pitch features and augmented with their delta features.

The classification in contrastive system 1 consisted of a Gaussian mean supervector representation of the segments obtained from the previous step through the adaptation of a Universal Background Model (UBM) with 256 components. Classification was performed employing a support vector machine (SVM) with a linear kernel. The feature vectors used in this classifier were 12 MFCCs plus energy as in the segmentation stage, augmented with their delta and delta-delta coefficients.

The contrastive system 2 used a classic GMM maximum likelihood classification with 512 components performed by doing MAP adaptation of a UBM with full-covariance matrices. The set of features was the same that was used in the primary system.

\subsubsection{Group 3}

Group 3 presented a single primary system based on three independent GMM-UBM detectors of broad acoustic classes (speech, music, and noise in every possible context) with a segmentation-by-classification strategy.

The system was based on MFCC feature vectors including shifted delta coefficients to capture the time dependency structure of the audio. Acoustic classes were modeled through 1024-component MAP-adapted GMMs. Each detector performed a frame-by-frame scoring obtaining one log likelihood stream per acoustic class. These score-streams were smoothed through an average filter over a sliding window in order to deal with the high variability of frame scores. Finally, the smoothed frame-level scores were independently calibrated for each acoustic class by means of linear logistic regression.

\subsubsection{Group 4}

Group 4 presented a primary system and a contrastive system with a segmentation-by-classification strategy for both of them.

The proposed system was based on a "binary key" (BK) modeling approach originally designed for speaker recognition [39] and later applied successfully in a speech activity detection task [40]. The approach provided a compact representation of a class model through a binary vector (vector only containing zeros and ones) by transforming the continuous acoustic space into a discrete binary one. This transformation was done by means of a UBMlike model called Binary Key Background Model (KBM). Once the binary representation of the input audio was obtained, subsequent operations were performed in the binary domain, and calculations mainly involve bit-wise operations between pairs of binary keys. Segment assignment was done by comparing each segment BK with the $\mathrm{N}$ BKs (previously estimated using the KBM and training data) for each of the $\mathrm{N}$ target audio classes. Two alternatives to compute the similarity between two binary keys were proposed, one for the primary system and other for the contrastive system, respectively.

\section{Experimental results}

This section presents and analyzes the results of the Albayzín-2014 Audio Segmentation Evaluation for all the primary and contrastive systems of each group.

Table 1 shows the segmentation error rate (as defined in Eq. 2) for the seven submitted systems. No system was trained with additional material apart from the audio provided for the evaluation. As can be seen from the table, both first (20.68 \%) and second (20.80\%) best 
Table 1 Segmentation error rate of participating systems

\begin{tabular}{lccc}
\hline & Primary & Contrastive 1 & Contrastive 2 \\
\hline Group 1 & 20.68 & - & - \\
Group 2 & 20.80 & 29.13 & 22.52 \\
Group 3 & 30.67 & - & - \\
Group 4 & 31.59 & 33.93 & - \\
\hline
\end{tabular}

systems obtained very similar performance even though the systems represent very different strategies to perform the segmentation: the primary system of group 1 is based on a fusion of two segmentation-by-classification approaches while the primary system of group 2 is based on a segmentation-and-classification approach. The primary systems of group 3 and group 4 also show similar performance (around $31 \%$ ), but they are far from the primary systems of groups 1 and 2. It is apparent that, for all groups, the primary systems outperform the contrastive systems, indicating that the choice of the main strategy of each group was done properly.

Figure 2 compares the misses (blue columns) and the false alarms (orange columns) of the participating systems for the overlapped acoustic classes individually (music, noise, and speech). Each system is represented by a $g X_{-} Y$ notation where $X$ indicates the group index and $Y$ indicates if the system is primary $(p)$ or contrastive $(c)$. The main source of the segmentation error comes from the noise detection, but the music detection also presents a considerably high error rate. This is because the music and noise classes are rarely presented alone but instead mixed with speech. Also, the lack of data for these isolated classes makes very difficult to train suitable models to detect them. Note that the two best systems (the primary systems of group 1 and group 2) have almost the same error rate coming from the detection of the noise class and both are much lower than those of the rest of the systems. The main difference between the systems submitted by groups 1 and 2 is that the former detects the music class better than the latter while the latter detects the speech classes slightly better than the former.
To accurately analyze the source of the errors, Fig. 3 presents the confusion matrices of the primary systems. The matrices show the percentage of the reference classes (rows) associated to hypothesized non-overlapping acoustic classes (columns). The classes are represented as SI for "silence," MU for "music," NO for "noise," SP for "speech," MN for "music+noise," SM for "speech+music," SN for "speech+noise", and SA for "speech+music+noise." The matrices clearly show that the most common errors are the confusions between "speech+music+noise" with "speech+noise" or "speech+music" and also between "speech+noise" and "speech." In addition, there is a common error in all the systems with "music+noise" being classified as "music." Note that the systems of group 2 and group 4 incur in a non-negligible error rate coming from the detection of the "silence" class since these systems do not implement a silence detector and, therefore, false alarms are produced.

A fusion of different systems usually improves the final result because the information comes from various sources [41]. For that purpose, the participants provided frame-level scores for each non-overlapping audio class for the training and test datasets. Table 2 shows the segmentation error rate when the scores of the primary systems are combined. The fusion was done with different combinations of the primary systems: group 1 and group 2 in the first row of the table; groups 1,2, and 3 in the second row; group 1, 2, and 4 in the third row; and a combination of all the systems in the fourth row of the table. We used a set of techniques to combine the scores. Firstly, one Gaussian distribution is estimated with class-dependent full covariance and mean with maximum likelihood on the training data for each class. This technique is known as Gaussian Back-End (GBE) and the results are shown in the first column of the table. We trained the fusion model with the scores computed over the training dataset, and we used the test dataset to compute the SER. To smooth the decisions, a Viterbi algorithm was chosen to determine the maximum likelihood transitions among classes. The segments are delimited by the transitions given by the Viterbi algorithm (second column of Table 2). On the
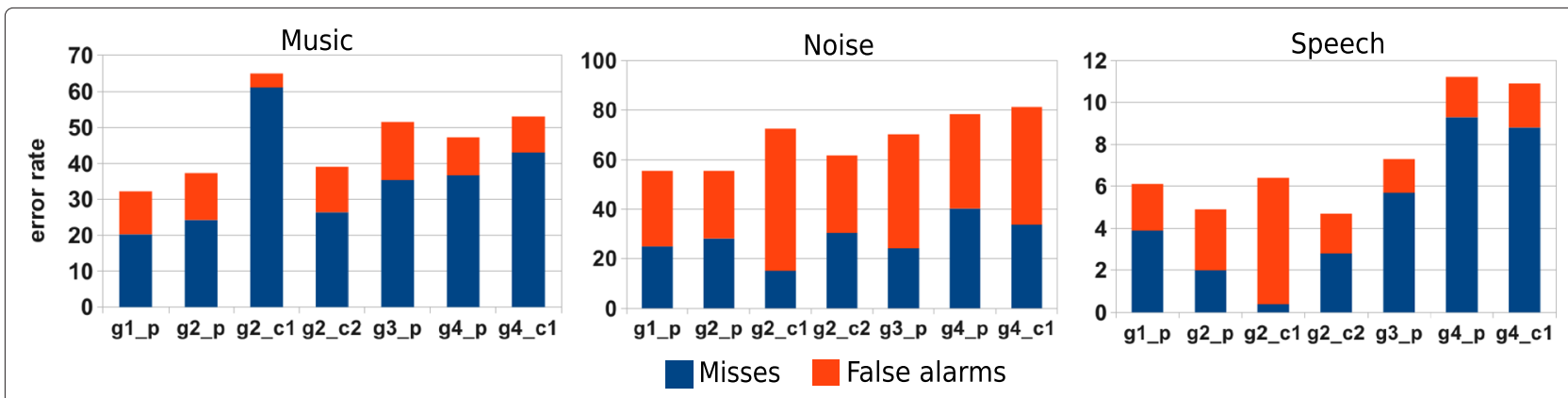

Fig. 2 Distribution of errors across the eight systems and for each acoustic class 


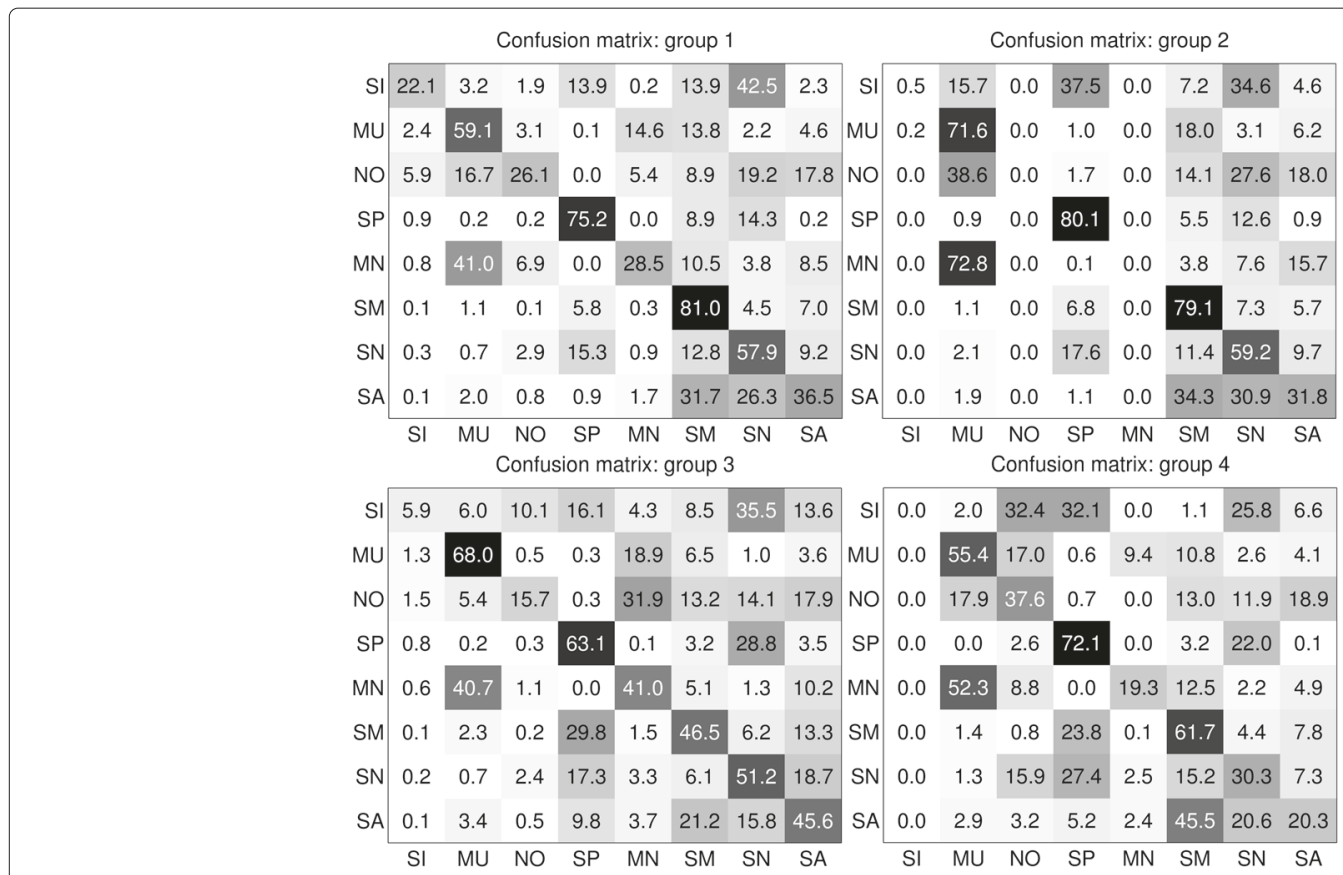

Fig. 3 Confusion matrices of the primary systems for the non-overlapped classes: SI (silence), MU (music), NO (Noise), SP (speech), MN (music and noise), SM (speech with music), SN (speech with noise), and SA (speech with music and noise)

other hand, we used the segments provided by the group 2 since the approach of this group is based on a previous unsupervised segmentation stage with BIC. In this case, we accumulated the log likelihood of each frame within the same segment, which was given the label of the class with the highest accumulated log likelihood. The results of this approach can be seen in the third column of the table. The best performance was attained when fusing the primary systems of groups 1,2 , and 3 . Finally, the last column of the table gives us an idea about the performance of the fusion if the segmentation was perfect. It clearly shows a degradation between 4 and $5 \%$ in the segmentation stage with regard to the oracle segmentation, because occasionally the delimitation boundaries among segments may be fuzzy. However, the fusion reduces the segmentation error rate for all the approaches compared with the winning primary system.

Table 2 Segmentation error rate of several score-level fusions of the primary systems. The result of the system $\mathrm{G} 1$ is 20.68 for comparison purposes

\begin{tabular}{ccccc}
\hline GBE & $\begin{array}{c}\text { GBE } \\
\text { Viterbi }\end{array}$ & $\begin{array}{c}\text { GBE } \\
\text { AccumLLk } \\
\text { GTM Seg. }\end{array}$ & $\begin{array}{c}\text { GBE } \\
\text { AccumLLk } \\
\text { Oracle Seg. }\end{array}$ \\
\hline G1+G2 & 19.60 & 19.41 & 19.30 & 14.36 \\
G1+G2+G3 & 19.56 & 19.31 & 19.16 & 14.30 \\
G1+G2+G4 & 19.94 & 19.77 & 19.64 & 15.58 \\
G1+G2+G3+G4 & 19.86 & 19.67 & 19.62 & 15.31 \\
\hline
\end{tabular}

\section{Conclusions}

This article presents the Albayzín-2014 Audio Segmentation Evaluation, including the main features of the database, an overview of the participating systems and evaluation and post-evaluation results. The new Albayzín2014 audio segmentation database combines data from two different media (TV and radio), with added noises of diverse nature, thus increasing the difficulty of the task. Using this database an audio segmentation task was proposed, where the systems were required to identify the presence of speech, music and/or noise, either isolated or overlapped. The Albayzín-2014 Audio Segmentation Evaluation contributed to the evolution of the audio segmentation technology in broadcast news domains by providing a more general and realistic database, compared to those used in the Albayzín-2010 and -2012 Audio Segmentation Evaluations [10,30]. The main features of the approaches and the results attained by seven segmentation systems from four different research groups have been presented and briefly analyzed. Three of the systems were based on a segmentation-and-classification strategy, while the rest of them were based on a segmentation-byclassification strategy.

Then, we presented seven segmentation systems and the results from four different research groups which participated in the Albayzín-2014 evaluation. The approaches and the results of each group were studied and compared. Three of the seven systems (from the same group) are based on a segmentation-and-classification strategy while 
the rest of the systems are based on a segmentation-byclassification strategy. Most of the systems used common speech recognition features, such as MFCC, LFCC, or PLP.

The two best systems attained a segmentation error rate (SER) of around $20 \%$, following two different strategies but with a common classification approach based on ivectors, showing the competitiveness of this technique. Both systems revealed that the main source of segmentation error was the detection of the noise class, mainly due to the low energy of noise signals. The results were analyzed using the non-overlapping classes through the confusion matrices of the primary systems. The matrices showed that the most common errors were the confusions between "speech+music+noise" with "speech+noise" or "speech+music" and also between "speech+noise" and "speech." Finally, the participating systems were combined under different approaches, yielding a relative improvement of up to $7.35 \%$ SER.

\section{Endnote}

${ }^{1}$ Spanish Thematic Network on Speech Technologies: http://www.rthabla.es.

\section{Abbreviations}

AC: acoustic classes; BN: broadcast news; HMM: hidden Markov model; GMM: Gaussian mixture model.

\section{Competing interests}

The authors declare that they have no competing interests.

\section{Acknowledgements}

This work has been partially funded by the Spanish Government and the European Union (FEDER) under the project TIN2011-28169-C05-02 and supported by the European Regional Development Fund and the Spanish Government ('SpeechTech4All Project' TEC2012-38939-C03).

\section{Author details}

${ }^{1}$ ViVoLab, Universidad de Zaragoza, Zaragoza, Spain. ${ }^{2}$ Aholab, Universidad de País Vasco, Bilbao, Spain. ${ }^{3}$ Multimedia Technologies Group (GTM), AtlanTIC Research Center, Universidade de Vigo, Vigo, Spain. ${ }^{4}$ ATVS, Universidad Autónoma de Madrid, Madrid, Spain. ${ }^{5}$ CAIAC, Universitat Autònoma de Barcelona, Barcelona, Spain

Received: 10 April 2015 Accepted: 16 November 2015

Published online: 01 December 2015

\section{References}

1. J Saunders, in 1996 IEEE International Conference on Acoustics, Speech, and Signal Processing Conference Proceedings. Real-time discrimination of broadcast speech/music, vol. 2 (IEEE Atlanta, 1996), pp. 993-996

2. E Scheirer, M Slaney, Construction and evaluation of a robust multifeature speech/music discriminator. 1997 IEEE International Conference on Acoustics, Speech, and Signal Processing. 2, 1331-1334 (1997)

3. S Srinivasan, D Petkovic, D Ponceleon, in Proceedings of the Seventh ACM International Conference on Multimedia. Towards robust features for classifying audio in the CueVideo system (ACM New York City, NY, 1999), pp. 393-400

4. S Kiranyaz, AF Qureshi, M Gabbouj, A generic audio classification and segmentation approach for multimedia indexing and retrieval. IEEE Trans. Audio Speech Lang. Process. 14(3), 1062-1081 (2006)

5. Z Huang, Y-c Cheng, K Li, V Hautamaki, C-h Lee, in Proc. Interspeech. A blind segmentation approach to acoustic event detection based on i-vector (ISCA Lyon, 2013), pp. 2282-2286
6. NIST, TREC NIST Evaluations. http://www.itl.nist.gov/iad/mig//tests/sdr/ Accessed 23 Nov 2015

7. S Galliano, E Geoffrois, D Mostefa, in Interspeech. The ESTER phase II evaluation campaign for the rich transcription of French broadcast news (ISCA Lisbon, 2005), pp. 3-6

8. J Zibert, F Mihelic, J Martens, H Meinedo, J Neto, L Docio, C Garcia-Mateo, P David, E Al, in Interspeech. The COST278 broadcast news segmentation and speaker clustering evaluation-overview, methodology, systems, results (ISCA Lisbon, 2005)

9. T Butko, CN Camprubí, H Schulz, in II Iberian SLTech. Albayzin-2010 audio segmentation evaluation: evaluation setup and results (FALA Vigo, 2010), pp. 305-308

10. A Ortega, D Castan, A Miguel, E Lleida, The Albayzin 2012 Audio Segmentation Evaluation (2012). http://dcastan.vivolab.es/wp-content/ papercite-data/pdf/ortega2012.pdf Accessed 23 Nov 2015

11. Tecnoparla, Tecnoparla Project. http://www.talp.upc.edu/tecnoparla

12. F Font, G Roma, X Serra, in Proceedings of the 21st ACM International Conference on Multimedia. Freesound technical demo (ACM Barcelona, Spain, 2013)

13. G Hu, 100 non-speech environmental sounds. http://www.cse.ohio-state. edu/dwang/pnl/corpus/HuCorpus.html Accessed 23 Nov 2015

14. NIST, The 2009 (RT-09) Rich transcription meeting recognition evaluation plan. http://www.itl.nist.gov/iad/mig/tests/rt/2009/docs/rt09-meetingeval-plan-v2.pdf Accessed 23 Nov 2015

15. Y Lavner, D Ruinskiy, A decision-tree-based algorithm for speech/music classification and segmentation. EURASIP J. Audio Speech Music Process. 2009, 1-15 (2009)

16. T Theodorou, I Mporas, N Fakotakis, An overview of automatic audio segmentation. I.J. Inf. Technol. Comput. Sci. 1, 1-9 (2014)

17. SImai, in IEEE International Conference on Acoustics, Speech, and Signal Processing Conference Proceedings. Cepstral analysis synthesis on the mel frequency scale (IEEE Boston, 1983), pp. 93-96

18. R Vergin, D O'Shaughnessy, V Gupta, in IEEE International Conference on Acoustics, Speech, and Signal Processing Conference Proceedings. Compensated mel frequency cepstrum coefficients, vol. 1 (IEEE Atlanta, 1996), pp. 323-326

19. R Vergin, Generalized mel frequency cepstral coefficients for large-vocabulary speaker-independent continuous-speech recognition. IEEE Trans. Speech and Audio Process. 7(5), 525-532 (1999)

20. E Wong, S Sridharan, in International Symposium on Intelligent Multimedia Video and Speech Processing. Comparison of linear prediction cepstrum coefficients and mel-frequency cepstrum coefficients for language identification (Kowloon Shangri-La Hong Kong, 2001), pp. 95-98

21. M Hasan, M Jamil, M Rahman, in International Conference on Computer and Electrical Engineering. Speaker identification using Mel frequency cepstral coefficients (Dhaka, 2004), pp. 28-30

22. P Dhanalakshmi, S Palanivel, V Ramalingam, Classification of audio signals using AANN and GMM. Appl. Soft Comput. 11(1), 716-723 (2011)

23. L Xie, W Fu, Z-H Feng, Y Luo, Pitch-density-based features and an SVM binary tree approach for multi-class audio classification in broadcast news. Multimedia Syst. 17(2), 101-112 (2011)

24. D Li, I Sethi, N Dimitrova, T McGee, Classification of general audio data for content-based retrieval. Elsevier, Pattern Recogn. Lett. 22, 533-544 (2001)

25. L Lu, H Zhang, $\mathrm{H}$ Jiang, Content analysis for audio classification and segmentation. IEEE Trans. Speech Audio Process. 10(7), 504-516 (2002)

26. TL Nwe, H Li, in IEEE International Conference on Acoustics, Speech and Signal Processing. Broadcast news segmentation by audio type analysis, vol. 2 (IEEE Philadelphia, 2005), p. 1065

27. A Hauptmann, R Baron, M Chen, in Proc. TRECVID. Informedia at TRECVID 2003: analyzing and searching broadcast news video (NIST Gaithersburg 2003)

28. S Dharanipragada, M Franz, Story segmentation and topic detection in the broadcast news domain. DARPA Broadcast News Workshop, 1-4 (1999). http://www.itl.nist.gov/iad/mig/publications/proceedings/ darpa99/html/abstract.htm Accessed 23 Nov 2015

29. A Gallardo-Antolín, J Montero, Histogram equalization-based features for speech, music, and song discrimination. Signal Process. Lett. 17(7), 659-662 (2010)

30. T Butko, C Nadeu, Audio segmentation of broadcast news in the Albayzin-2010 evaluation: overview, results, and discussion. EURASIP J. Audio Speech Music Process. 2011(1), 1 (2011) 
31. M Markaki, Y Stylianou, Discrimination of speech from nonspeech in broadcast news based on modulation frequency features. Speech Commun. 53(5), 726-735 (2011)

32. R Huang, J Hansen, Advances in unsupervised audio classification and segmentation for the broadcast news and NGSW corpora. IEEE Trans. Audio Speech Lang. Process. 14(3), 907-919 (2006)

33. SS Chen, PS Gopalakrishnan, in Proc. DARPA Broadcast News Workshop. Speaker, environment and channel change detection and clustering via the Bayesian information criterion (Lansdowne, 1998)

34. $Y W u, C-h$ Chiu, Automatic segmentation and identification of mixed-language speech using delta-BIC and LSA-based GMMs. IEEE Trans. Audio Speech Lang. Process. 14(1), 266-276 (2006)

35. M Kotti, E Benetos, C Kotropoulos, Computationally efficient and robust BIC-based speaker segmentation. IEEE Trans. Audio Speech Lang. Process. 16(5), 920-933 (2008)

36. A Misra, in Proc. Interspeech. Speech/Nonspeech Segmentation in Web Videos (ISCA Portland, 2012)

37. D Castán, A Ortega, A Miguel, E Lleida, Audio segmentation-byclassification approach based on factor analysis in broadcast news domain. EURASIP J. Audio Speech Music Process. 34, 1-13 (2014)

38. A Mesa, JLN Ortega, A Teixeira, EH Pérez, PQ Morales, AR Garcia, IG Moreno, IberSPEECH 2014: VIII Jornadas en Tecnologías del Habla and IV Iberian SLTech Workshop (2014). http://iberspeech2014.ulpgc.es/index. php/online Accessed 23 Nov 2015

39. X Anguera, J Bonastre, in INTERSPEECH 2010, 11th Annual Conference of the International Speech Communication Association, Makuhari, Chiba, Japan, September 26-30, 2010. A novel speaker binary key derived from anchor models (ISCA Makuhari, Chiba, Japan, 2010), pp. 2118-2121

40. H Delgado, C Fredouille, I Serrano, in INTERSPEECH 2014, 15th Annual Conference of the International Speech Communication Association, Singapore, September 14-18, 2014. Towards a complete binary key system for the speaker diarization task (ISCA Singapore, 2014), pp. 572-576

41. J Kittler, Combining classifiers: A theoretical framework. Pattern Anal. Appl. 1(1), 18-27 (1998)

\section{Submit your manuscript to a SpringerOpen ${ }^{\circ}$ journal and benefit from:}

- Convenient online submission

- Rigorous peer review

- Immediate publication on acceptance

- Open access: articles freely available online

- High visibility within the field

- Retaining the copyright to your article 